\title{
Stochastic Boundary, Diffusion, Emittance Growth and Lifetime calculation for the RHIC e-lens
}

\author{
N. P. Abreu, W. Fischer, Y. Luo, G. Robert-Demolaize
}

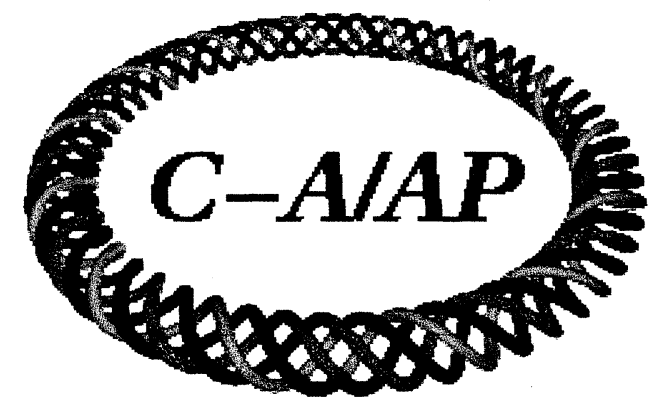

Collider-Accelerator Department

Brookhaven National Laboratory

Upton, NY 11973

Notice: This document has been authorized by employees of Brookhaven Science Associates, LLC under Contract No. DE-AC02-98CH10886 with the U.S. Department of Energy. The United States Government retains a nonexclusive, paid-up, irrevocable, world-wide license to publish or reproduce the published form of this document, or allow others to do so, for United States Government purposes. 
C-AD $/ \mathrm{AP} / 347$

\title{
Stochastic Boundary, Diffusion, Emittance Growth and Lifetime calculation for the RHIC e-lens
}

\author{
N.P. Abreu, W. Fischer, Y. Luo and G. Robert-Demolaize
}

January 8, 2009

\begin{abstract}
To compensate the large tune shift and tune spread generated by the head-on beam-beam interactions in polarized proton operation in the Relativistic Heavy Ion Collider (RHIC), a low energy electron beam with proper Gaussian transverse profiles was proposed to collide head-on with the proton beam. In this article, using a modified version of SixTrack [1], we investigate stability of the single particle in the presence of head-on beam-beam compensation. The Lyapunov exponent and action diffusion are calculated and compared between the cases without and with beam-beam compensation for two different working points and various bunch intensities. Using the action diffusion results the emittance growth rate and lifetime of the proton beam is also estimated for the different scenarios.
\end{abstract}

\section{Introduction}

To maintain the beam lifetime and polarization in the polarized proton operation in the Relativistic Heavy Ion Collider (RHIC) the current working points for the proton beams are $(28.685,29.695)$ and $(28.695,29.685)$ for the two RHIC rings, which are constrained between $2 / 3$ and $7 / 10$ [2] betatron resonances lines. It has been shown by both experiments and simulations that when the fractional betatron tune is close to $2 / 3$ the beam lifetime is strongly affected and when the vertical tune is close to $7 / 10$, both the luminosity lifetime and the proton polarization are worse. In the 2008 RHIC pp run, the bunch intensity had reached about $1.5 \times 10^{11}$ protons, however to increase the bunch intensity beyond $2.0 \times 10^{11}$ it will be increasingly difficult to accommodate the beam-beam generated tune spread between $2 / 3$ and $7 / 10$ resonances.

One solution is to adopt head-on beam-beam compensation [3, 4]. In the Tevatron at Fermi National Accelerator Laboratory (Fermilab), a low energy electron beam (elens) has been used to compensate the tune variations in the proton bunch train [5]. Experimentally the long-range beam-beam compensation does increase the lifetime of PACMAN bunches in the bunch trains [6]. In our study, we investigate whether a device like the Tevatron e-lens can be used to mitigate the head-on beam-beam effects in RHIC.

To evaluate the benefits and side effects from the head-on beam-beam compensation with e-lens in RHIC, detailed simulation studies have to be done. In 2005, preliminary simulation studies did show that e-lenses in the RHIC rings will greatly reduce the tune shift and tune spread generated by the p-p head-on beam-beam interactions. However, more careful studies also have to be carried out to evaluate its impacts on the lifetime and emittance evolution of the proton beams $[7,8]$. 
In this article, we report the results from the study of stability of a single proton in the presence of head-on beam-beam compensation in RHIC. We will first introduce the parameters of the proton and electron beams and the lattice for this study, followed by the beam-beam interaction model and tracking code we adopt. Then, we calculate and compare the Lyapunov exponent and the action diffusion and compare the results between the cases without and with head-on beam-beam compensations. With the diffusion results we estimate the emittance growth rate and the lifetime of the proton beam with different bunch intensities and also with and without the e-lens.

\section{Beam parameters}

For the RHIC pp run, the two proton beams collide at IP6 and IP8. The proton beam in the Blue ring circulates clockwise, while the proton beam in the Yellow ring circulates counter-clockwise. In the current design, the RHIC e-lenses are close to the interaction point IP10. Two e-lenses are needed for the RHIC head-on beam-beam compensation scheme, one for the Blue ring and another one for the Yellow ring and in this setup the two proton beams are vertically separated in the interaction region (IR) of IP10. Each e-lens, for the Yellow and Blue rings, are assumed to be $2 \mathrm{~m}$ long and are symmetrically placed 1.5 meter away from IP10. However, in the following simulations, for simplicity, we assume the e-lenses are exactly located at IP10 [4].

The proton beam parameters used in this study are show in Tab. 1. The linear chromaticities are corrected to $Q_{x, y}^{\prime}=+1$ and the multipole magnetic field errors in the triplet quadrupoles and separation dipole magnets in the IRs are included in the lattice. The bunch intensity is varied between $N_{p}=1.3 \times 10^{11}$, which was the intensity during the $2006 \mathrm{p}-\mathrm{p}$ run, and $N_{p}=3.0 \times 10^{11}$ protons/bunch.

In this study we assume that the electron beam has the same transverse Gaussian profile as the proton beam at IP10 and, for the full head-on beam-beam compensation the electron particle density is twice of that of the proton bunch intensity, while for the half head-on beam-beam compensation the electron particle intensity is the same as the proton intensity per bunch.

\section{Simulation Code}

In our study, the 6-D simplectic tracking code SixTrack [1] is used. In this code, the linear elements are treated as thick elements while the nonlinear elements are treated as thin lenses. The beam-beam interaction calculation is based on weak-strong model. SixTrack has an internal limitation on the particle number and in order to be able to simulate with an arbitrary number of particles at a time the source code was modified so that a distribution file can be used as the initial particles coordinate input. This feature is achieved by applying a DO loop of the 64 pack which extended the maximum number of particles to 20000 and to activate this special simulation condition the lhc flag in the BEAM-BEAM element has to be set to 9. An output file is also generated, when the 
Table 1: Parameters for the proton beams

\begin{tabular}{lcc}
\hline \hline quantity & unit & value \\
\hline lattice & $\mathrm{m}$ & 3833.8451 \\
ring circumference & $\mathrm{GeV}$ & 250 \\
energy & - & 266 \\
relativistic $\gamma$ & - & $\mathrm{IP} 6, \mathrm{IP} 8$ \\
beam-beam collision points & - & $\mathrm{IP} 10$ \\
beam-beam compensation point & $\mathrm{m}$ & 0.5 \\
$\beta_{x, y}^{*}$ at IP6 and IP8 & $\mathrm{m}$ & 10 \\
$\beta_{x, y}^{e}$ at IP10 & $\mathrm{m}$ & 10 \\
$\beta_{x, y}^{*}$ at all other IPs & $\mathrm{rad}$ & $(10.6 \pi, 8.6 \pi)$ \\
phase advance between IP6 and IP8 $\left(\mu_{x}, \mu_{y}\right)$ & $\mathrm{rad}$ & $(8.4 \pi, 10.9 \pi)$ \\
phase advance between IP8 and IP10 $\left(\mu_{x}, \mu_{y}\right)$ & & \\
proton beam & - & $2 \times 10^{11}$ \\
particles per bunch $N_{p}$ & $\mathrm{~mm} \cdot \mathrm{mrad}$ & 2.5 \\
normalized transverse rms emittance $\epsilon_{x, y}$ & $\mathrm{~mm}$ & 0.068 \\
transverse rms beam size at collision points $\sigma_{x, y}^{*}$ & $\mathrm{~mm}$ & 0.31 \\
transverse rms beam size at e-lens $\sigma_{x, y}^{e}$ & - & $(28.695,29.685) /(28.685,29.695)$ \\
transverse tunes $\left(Q_{x}, Q_{y}\right)$ & - & $(1,1)$ \\
chromaticities $\left(Q_{x}^{\prime}, Q_{y}^{\prime}\right)$ & - & -0.01 \\
beam-beam parameter per IP $\xi_{\mathrm{p} \rightarrow \mathrm{p}}$ & & \\
\hline longitudinal parameters & - & 360 \\
harmonic number & $\mathrm{kV}$ & 300 \\
rf cavity voltage & $\mathrm{eV} \cdot \mathrm{s}$ & 0.17 \\
rms longitudinal bunch area & - & $0.14 \times 10^{-3}$ \\
rms momentum spread & $\mathrm{m}$ & 0.44 \\
rms bunch length & & \\
\hline \hline
\end{tabular}



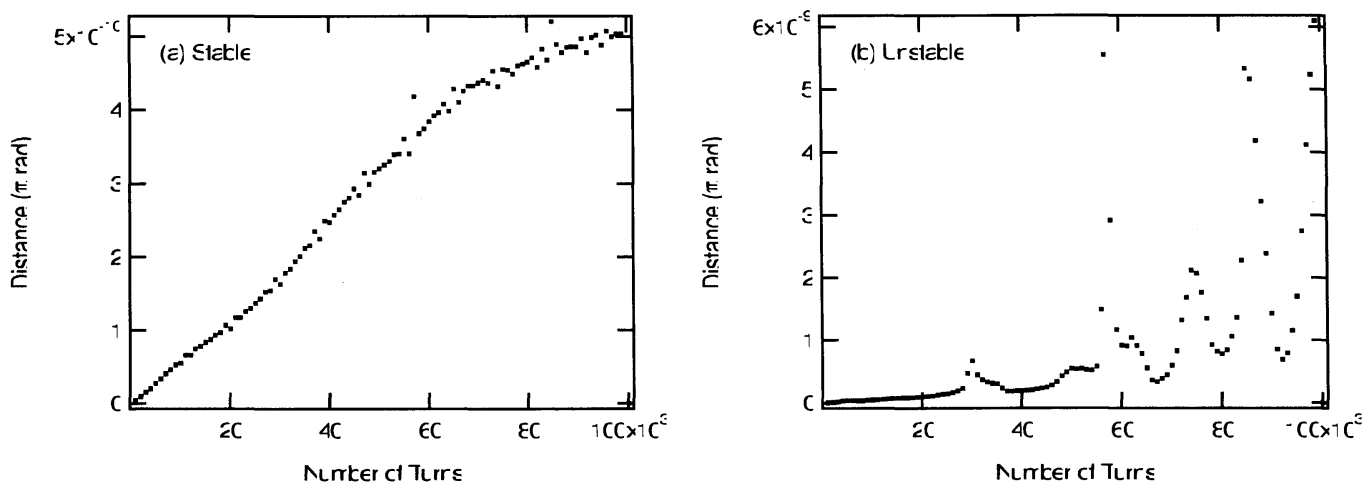

Figure 1: Distance of the twin particles for (a) a regular motion and (b) a chaotic pair.

external distribution is used, that contains the $6 \mathrm{D}\left(x, x^{\prime}, y, y^{\prime}, s, \Delta p / p\right)$ parameters for each particle in each turn.

\section{Stochastic Boundary}

In order to support the emittance simulations/calculation we investigated the stochastic boundary of the system. We used two particles with a small difference in the initial position in the $6 \mathrm{D}$ phase space and track both for $10^{6}$ turns, at every 1000 turns the distance in phase space is calculated in the post processing routine of SixTrack. An example of the simulation result is in Fig. 3 which show a plot of the phase space distance calculated for: (a) a stable particle and (b) a chaotic particle. In the first case the angular distance of the particles increase linearly with the number of turns and for the second case this growth is exponential at the last turns revealing the chaoticity of the particle motion.

In Figures 2 and 3 are the results of the tracking for the two working points studied for on momentum (synchronous) particles, the case of off momentum particles does not have any particular difference. With these simulations we do not observe stability any extended space. The particles labeled stable in the plots means that up to $10^{6}$ turns their trajectories are regular. The particles represented by red dots have chaotic trajectories and it is possible to observe that almost all the particles, including the core ones, are chaotic. The fact that there is no regular motion indicates that the core particles experience diffusion and that emittance growth should be observed in the simulations. The use of the head-on compensation does not result in an improvement in the chaotic behavior of the particles. 

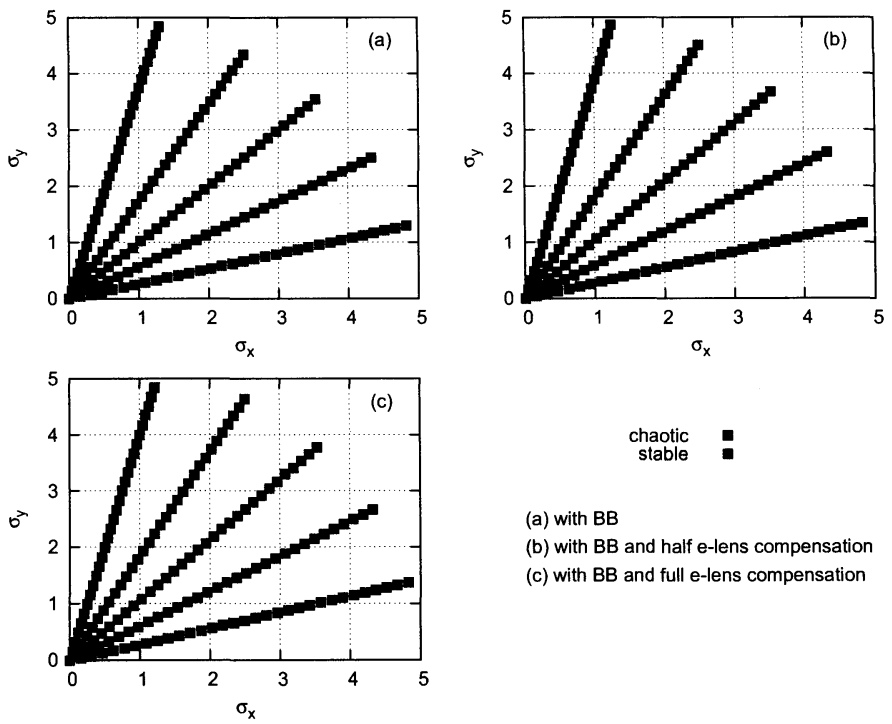

Figure 2: Chaoticity for particles for the working point above the diagonal $(0.685,0.695)$. All three plots show that almost all particles up to $5 \sigma$ are chaotic.
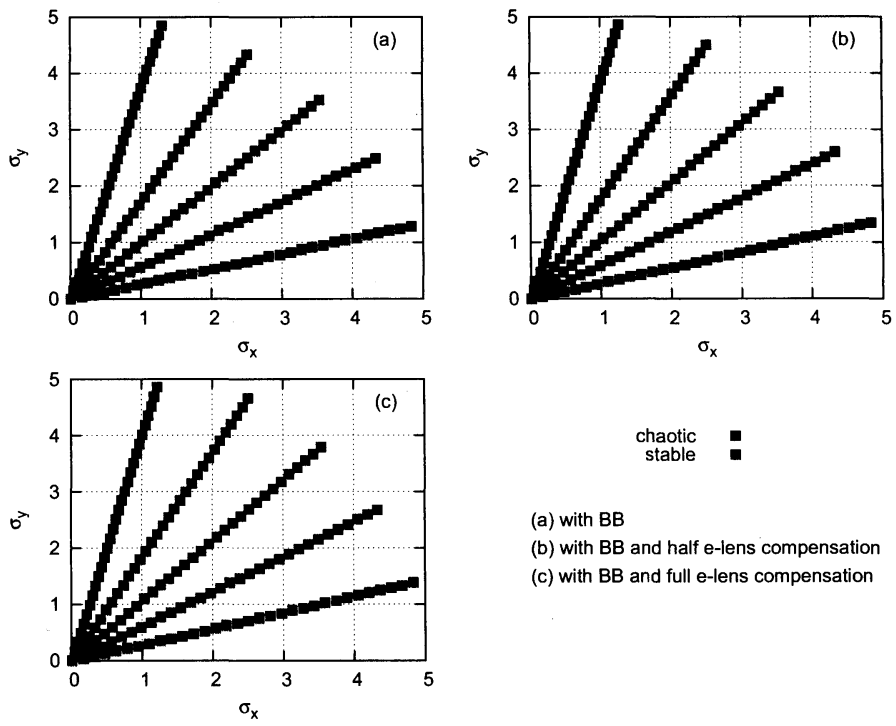

(a) with $\mathrm{BB}$

(b) with BB and half e-lens compensation

(c) with $\mathrm{BB}$ and full e-lens compensation

Figure 3: Chaoticity for particles for the working point bellow the diagonal $(0.695,0.685)$. All three plots show that almost all particles up to $5 \sigma$ are chaotic. 


\section{Diffusion Simulations and Emittance Growth}

Emittance growth due to the non-linearities of the beam-beam interaction is a major problem in hadron colliders. Random fluctuations of the tune, closed orbit and beam size are also some of the of the possible effects that can cause emittance growth especially for tunes close to strong resonance lines.

In order to estimate the emittance growth rate first it is necessary to have the particles diffuse as a function of the initial amplitude. We used SixTrack to calculate the trajectories of 64 particles with the same initial action for $5 \times 10^{4}$ turns and for each turn we calculate the rms spread $\left(\sigma_{J}\right)$ of the action for all the particles. We then average the rms action values over 500 turns to eliminate the short-term fluctuations due to phase-space orbit deviations form the linear model [9] that can be caused by weak resonances.

The diffusion coefficient is defined as

$$
D(J)=\lim _{N \rightarrow \infty} \frac{\langle J(N)-J(0)\rangle^{2}}{N}=\lim _{N \rightarrow \infty} \frac{\sigma_{J}^{2}(N)}{N}
$$

and in order to calculate its values we fit a line $\left(\sigma_{J}^{2}(N)=a+b N\right)$ over the rms action values for 10 points spaced by 5000 turns, so that we have $D(J)=b$. Once the diffusion points are calculated we fit the result using the expression $D(J)=A J+B J^{2} \exp (C J)$. This fitting function behaves like a parabola for the inner-most particles in the bunch which is in agreement with calculation for diffusion due to beam-beam effects [10]; and for the tails it has a exponential behavior to account for diffusion due to the lattice non-linearities.

Assuming that the diffusion in action is a Markov process and that the drift coefficient is half of the derivative of the diffusion coefficient (this is true for most Hamiltonan systems), we can describe the evolution of the phase-space distribution $\rho(J, t)$ by a Fokker-Planck equation

$$
\frac{\partial \rho}{\partial t}=\frac{1}{2} \frac{\partial}{\partial J}\left(D(J) \frac{\partial \rho}{\partial J}\right)
$$

and the using that $[10]$

$$
\langle J(t)\rangle=\frac{\int_{0}^{J_{c u t}} J \rho(t, J) d J}{\int_{0}^{J_{c u t}} \rho(t, J) d J}
$$

where $J_{\text {cut }}$ is a cut equivalent to $99.5 \%$ emittance $(3 \sigma)$. Assuming that the phase-space distribution is gaussian in the $\left(x, x^{\prime}\right)$ space and therefore an exponential in action

$$
\rho(J, t) \propto \exp \left(-J(t) / 2 \varepsilon_{0}\right)
$$

where $\varepsilon_{0}$ is the initial emittance, the emittance growth rate in first approximation can be calculated as

$$
\left\langle\frac{d \varepsilon}{d t}\right\rangle=\frac{1}{2} \frac{\int_{0}^{J_{c u t}} D(J) \frac{\partial \rho(J, t)}{\partial J} d J}{\int_{0}^{J_{c u t}} \rho(J, t) d J}=\frac{1}{4 \varepsilon_{0}} \frac{\int_{0}^{J_{c u t}} D(J) \rho(J, t) d J}{\int_{0}^{J_{c u t}} \rho(J, t) d J}
$$

assuming that the derivative of the density function for $J>J_{\text {cut }}$ are close to zero, this is, the derivative $\frac{\partial \rho(J, t)}{\partial J} \approx 0$ at the tails of the distribution. 

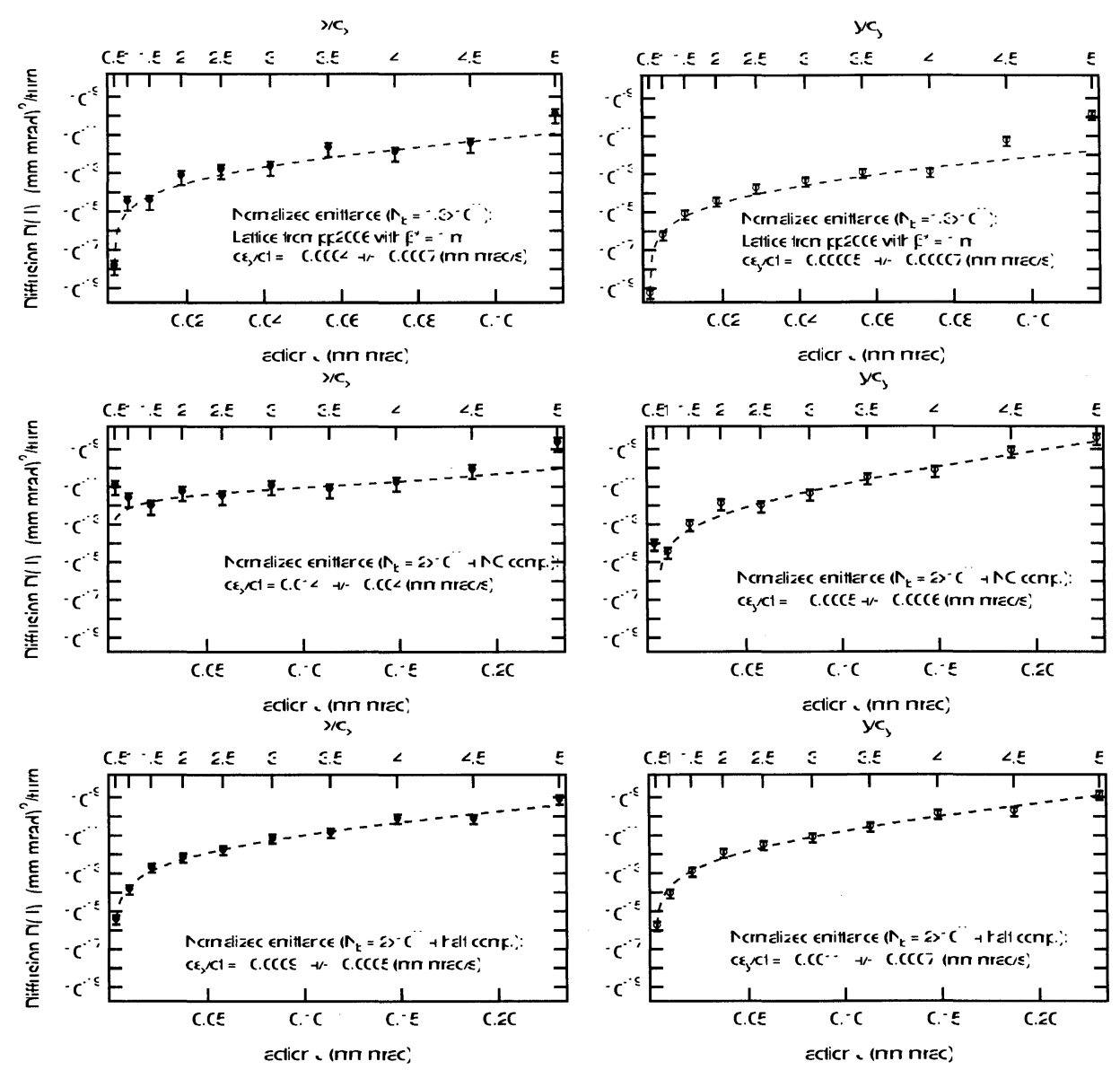

Figure 4: Example of the diffusion simulation results and the fitting for the working point above the diagonal.

Some results for the diffusion simulation and the fitting are shown in Fig.4 for the working point above the diagonal, on Figs. 5 and 6 there are all the fittings of the diffusion coefficient for different bunch intensities and working points. In each plot, of Fig.4, the label shows the calculated normalized emittance growth for RHIC $\left(\varepsilon_{N}=\gamma 6 \sigma^{2} / \beta\right)$. The measured emittance growth rate for the proton run of 2006 is about $2 \times 10^{-4}(\mathrm{~mm} \mathrm{mrad})^{2} / \mathrm{s}$ for a bunch intensity of $1.3 \times 10^{11}$ protons, and as can be notice the values which come from the simulation are close to the measured one considering the same bunch intensities.

Fig. 7 shows the emittance growth rate calculated for various configurations (different bunch intensity and no, half and full beam-beam compensation). The growth rate for the case of the working point above the diagonal with bunch intensities of $N_{b}=2-3 \times 10^{11}$ and no compensation reflects the fact that at this intensities the horizontal tune is close or crosses the $2 / 3$ resonance which is known to deteriorate the beam quality, once the beam- 


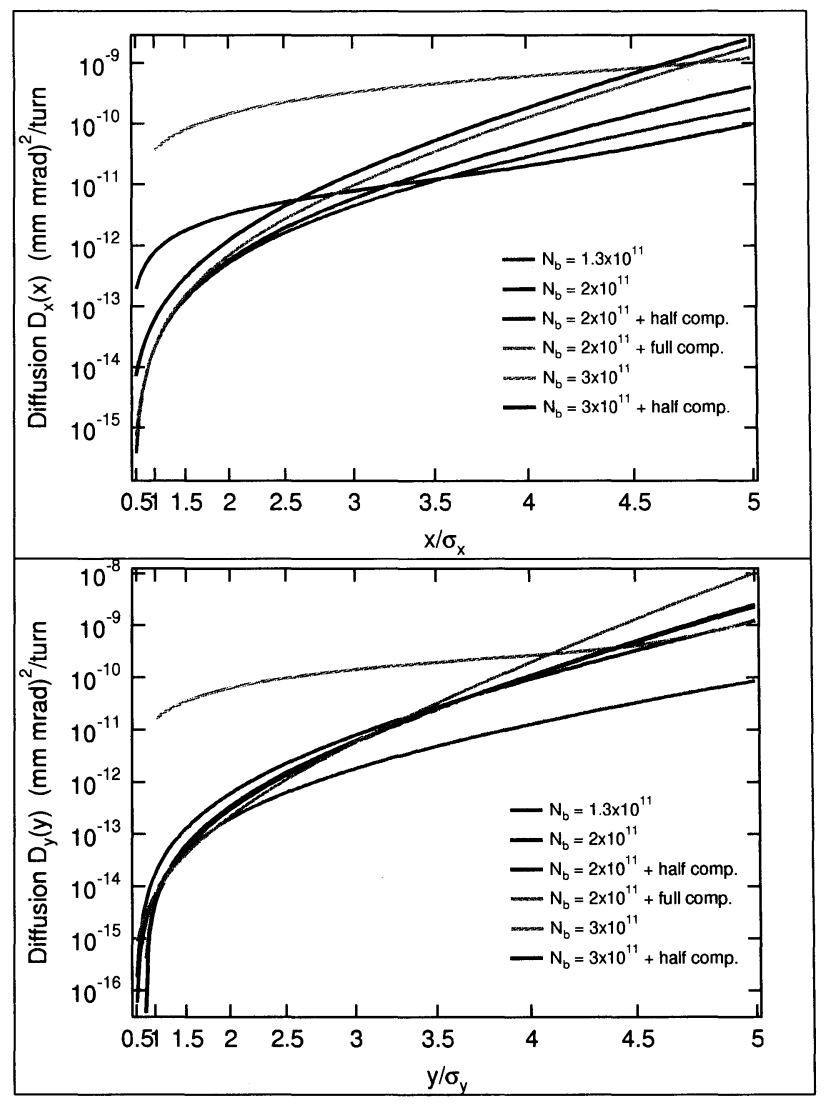

Figure 5: Example of the diffusion simulation results and the fitting for the working point above the diagonal.

beam head-on compensation in turned on the emittance growth rate is reduced to a value comparable to the one measured in 2006 since there is no crossing of the $2 / 3$ line anymore. For the case with the tunes below the diagonal the emittance growth for the case with the highest bunch intensities and no compensation the same resonance is crossed but this time in the vertical plane leading to an increase in the emittance growth rate as well however it appears that the vertical crossing of this resonance is not as strong as the horizontal. The configuration with the lowest bunch intensity (left most side of the plots in Fig.7) and the cases with compensation (right most side of the plots in Fig.7) show that, within the error, the emittance growth rates are the same which show that the compensation brings down the growth rate to values which were already shown experimentally to be acceptable during the polarized proton run of 2006 and 2008. The drawback of this calculation is that although the values are close the error bars are large, due to the difficulty to calculate the diffusion from the simulation results, and so the accuracy of the comparison in within a factor of $2-3$. 


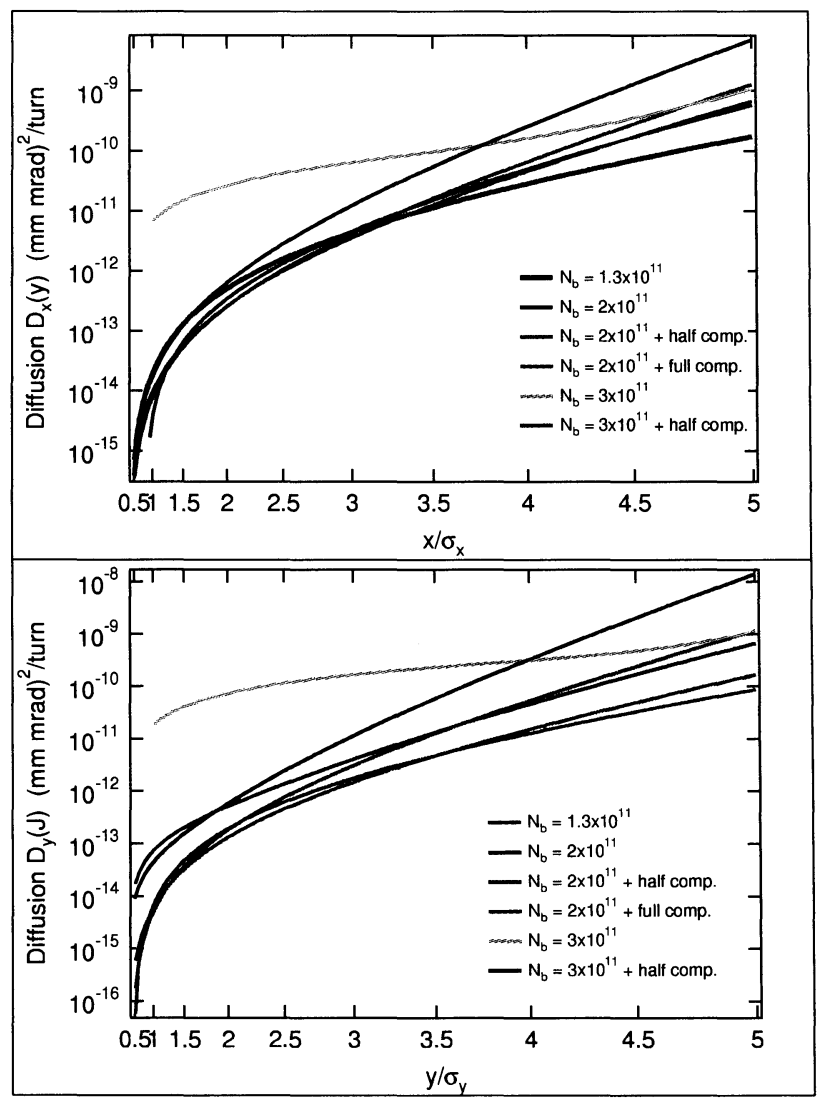

Figure 6: Example of the diffusion simulation results and the fitting for the working point below the diagonal. 

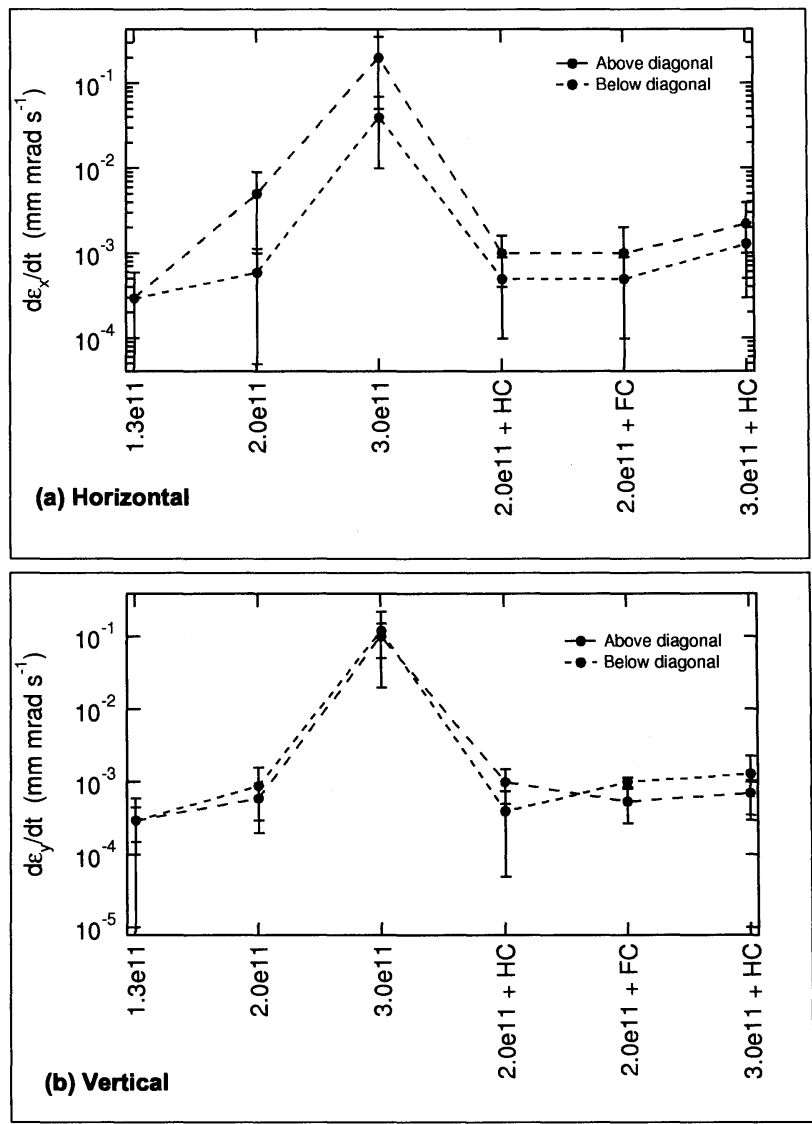

Figure 7: Example of the diffusion simulation results and the fitting for the working point above the diagonal. 


\section{Diffusion Simulations and Lifetime Calculation}

From Figs. 5 and 6 it is possible to observe that even though the compensation improves the diffusion at the core it often increases the diffusion for particles beyond $4 \sigma$ a behavior also seen in other studies [12]. In this section, using the results from the diffusion simulation we are going to calculate the lifetime of the proton beam and try to verify that the increase of the diffusion in the tails does not impose a limitation to the use of the e-lens as a BB head-on compensator.

We are going to use the same method of lifetime calculation as described in [11], which describes the lifetime calculation using the diffusion coefficients simulated for a given set of beam parameters (as intensity, emittance, etc..).

Using the diffusion fitted in the previous section and solving the diffusion equation for each plane we can get the impact of the head-on compensator on the lifetime of the proton beam, show in Fig 8. Note that even though the diffusion for particles beyond $4 \sigma$ is greater with the use of the e-lens the impact on the lifetime is rather small for the case of $2 \times 10^{11}$ protons/bunch. On the other hand, for the case with $3 \times 10^{11}$ protons/bunch the lifetime is greatly improved when the compensation is on.

Those results, however, show only an overall trend to the lifetime since the diffusion coefficients have a great uncertainty not much can be said about the effect of the elens compensation in the $2 \times 10^{11}$ protons/bunch for example. Another important feature, not taken it account into the calculation is the fact that the e-lens compensation depends on the phase advance between IPs and since we a tracking with the normal lattice the phase advance between IPs are far from the ideal one (see Tab. 1), which should be $90^{\circ}$ for the total compensation of at least one of the IPs head-on proton collision.

\section{Conclusions}

In our beam-beam simulations, without and with an electron lens, we find that almost all particles are chaotic, even those in the beam core. Based on this observation we investigated the emittance growth by calculating the diffusion coefficient at selected phase space locations. From the diffusion calculation we observe that the electron lens is able to almost restore the diffusion levels at the core however at the cost of increasing the diffusion for particles beyond $4 \sigma$. From the emittance growth rate calculation this reduction of the diffusion in the beam core appears as a reduction in the emittance growth rate calculated using the diffusion results for each working point, however the error bars, due to the difficulty to have an accurate value for the diffusion coefficient, are too large to enable a close comparison between the cases with lower growth rate. Also using the diffusion coefficients we calculated the beam lifetime for each case, showing that there is no great improvement for the lower intensity case $\left(2 \times 10^{11}\right.$ protons /bunch $)$ but a great improvement for the higher intensities $\left(3 \times 10^{11}\right.$ protons /bunch). It is necessary further investigation in the dependence of the lifetime on beam and lattice parameters as chromaticities and phase advances, which are not covered in this report. In all cases we 

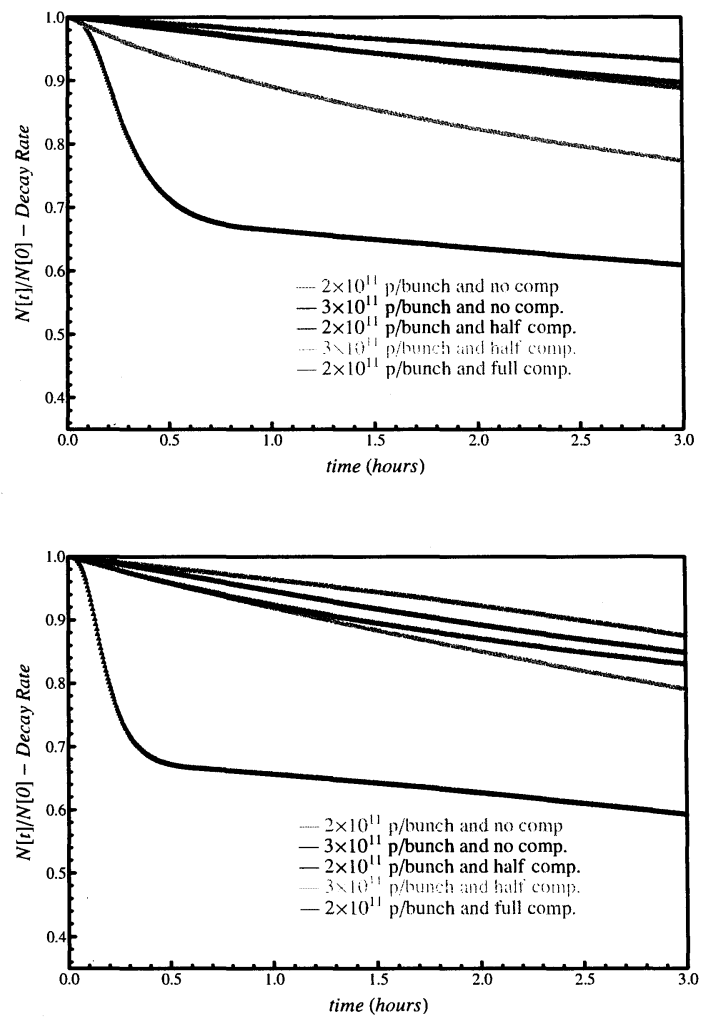

Figure 8: Calculated lifetime for different configurations with and without compensation and for the two working points in RHIC. Working points: top) $(0.685,0.695)$ and bottom $)(0.695,0.685)$. The use of head-on compensation does not cause a substantial reduction in lifetime even though the diffusion for high action particles is usually bigger in this case. For a bunch intensity of $2 \times 10^{11}$ protons /bunch there is not much change in the lifetime with compensation however for the higher intensity case $\left(3 \times 10^{11}\right)$ the improvement in lifetime is visible. 
used an ideal electron lens that had the same transverse profile as the proton beam, is not affected by the proton beam, and has no position or current errors.

\section{References}

[1] F. Schmidt, "SixTrack Manual", Version 4.1, CERN/SL/94-56 (AP). Availbale at http://frs.home.cern.ch/frs/

[2] W. Fischer, Beam-beam and BTF, 2006 RHIC Accelerator Physics Experiments Workshop, November 2-3, 2005, BNL.

[3] V. Shiltsev, Electron lenses in Tevatron, RHIC and LHC, in the 2005 RHIC APEX workshop, BNL, November 2005.

[4] Y. Luo, W. Fischer, Outline of using an electron lens for the RHIC head-on beam-beam compensation, BNL C-AD AP Note 286, July 2007.

[5] V. Shiltsev, V. Danilov, D. Finley, and A. Sery, Phys. Rev. ST Accel. Beams 2, 071001 (1999).

[6] V. Shiltsev, Y. Alexahin, K. Bishofberger, V. Kamerdzhiev, G. Kuznetsov, and X.-L. Zhang, Phys. Rev. Lett. 99, 244801 (2007).

[7] Y. Luo, RHIC Head-On Beam-Beam Compensation with e-lens, in the LARP miniworkshop on beam-bea, compensation, SLAC, July 2007.

[8] Y. Luo, Progress in e-lens work for RHIC, in the LARP 9th collaboration meeting, SLAC, October 2007.

[9] J. Irwin, "Diffusive losses from SSC particle bunches due to long-range beam-beam interactions", SSC-233, September 1989.

[10] T. Sen, J. A. Ellison, Phys. Rev. Lett. vol. 77, number 6 page 1051.

[11] N.P. Abreu, W. Fischer, Y. Luo and G. Robert-Demolaize, Difusion Simulation and Lifetime Calculation for RHIC, C-A/AP/346.

[12] Y. Luo, W. Fisher and N. P. Abreu, Stability of single particle motion with head-on beam-beam compensation in the RHIC, $C$-A/AP/310.

[13] N. P. Abreu, W. Fischer, Y. Luo and G. Robert-Demolaize, The effect of head-on beam-beam compensation on the stochastic boundaries and particle diffusion in RHIC, European Particle Accelerator Conference 2008, Genoa, Italy. 